\title{
The changing profile of infantile tremor syndrome in hilly terrain of India
}

\author{
Deeksha A. Singla, Milap Sharma, Seema Sharma, Vipin Sharma ${ }^{1}$ \\ Departments of Pediatrics and ${ }^{1}$ Orthopedics, Dr. Rajendra Prasad Government Medical College and Hospital, Tanda, Himachal Pradesh, India
}

\section{ABSTRACT}

Background: Infantile tremor syndrome (ITS) is characterized by anemia, skin pigmentation, tremors, physical, and mental regression without a defined etiopathogenesis and low incidence. Materials and Methods: We have studied 9 patients over 1 year for the changing clinical and laboratory variables of patients with ITS. Neuroregression and anemia were presented in all followed by tremors in 5 and hypotonia in 2. Result: Sepsis screen was positive in 6 and urine cultures in 2. Antibiotics were required in 6. ITS with changing parameters still significantly contributes to healthcare burden. Conclusion: It is important to screen for urinary infection and septicemia to avoid antibiotic abuse.

Key words: Anemia, infantile, lethargy, regression, tremors, urinary tract infection

\section{Introduction}

Infantile tremor syndrome (ITS) is a syndrome characterized by anemia, skin pigmentation, tremors, physical, and mental regression..$^{[1-5]}$ It has been reported in children between 5 months and 3 years of age with a male predominance. The exact incidence of ITS is not available. However, in various studies $0.87-1.55 \%$ hospital admissions were due to ITS. ${ }^{[1]}$ The exact etiopathogenesis of this condition remains obscure. The purpose of this case series was to determine factors responsible for its continuing prevalence despite better knowledge and weaning practices.

\section{Case Report}

We have studied 9 patients presenting to us with typical features of ITS and pre-ITS ${ }^{[6]}$ in a tertiary care hospital of hilly terrain [Table 1]. A detailed history and examination were carried out in each patient followed

\begin{tabular}{|l|l|}
\hline \multicolumn{2}{|c|}{ Access this article online } \\
\hline Quick Response Code: & Website: \\
\hline & www.ruralneuropractice.com \\
\cline { 2 - 3 } & \\
\hline
\end{tabular}

by investigations. Investigations included complete hemogram, peripheral smear to type the anemia and malaria parasite, serum electrolytes, renal and liver function tests along with sepsis screen, and urine routine examination. Blood and urine cultures, serum B12 levels, chest X-ray, and tuberculosis screen were done when indicated. Written informed consent was taken from all. Resources available at the hospital were utilized for the same.

Total 9 patients, 3 males and 6 females presented over the period of 1 year. The mean age of presentation was 13.5 months, ranging from 7 months to 18 months. All $(100 \%)$ patients admitted with the complaint of lethargy and weakness with a mean duration of 21 days. Neuroregression was present in all $(100 \%)$ with a mean delay of 7 months ranging from 4 to 11 months in all spheres of development. Tremors were present in 5 (55\%) and hypotonia in $2(22 \%)$ reflecting neurological changes. All $(100 \%)$ were healthy term babies born by normal vaginal delivery. History of faulty feeding was present in all. Seven (78\%) were exclusively breastfed and $2(22 \%)$ improperly weaned with diluted milk. Protein energy malnutrition was present in $5(55 \%)$ out of which $2(40 \%)$ had Grade I and 3 had Grade II (60\%) protein energy malnutrition as per IAP classification. Three (33\%) had severe wasting according to WHO criteria. There was no stunting in any patient. OFC records of all patients were within normal range for their age [Table 2].

Address for correspondence:

Dr. Seema Sharma, Department of Pediatrics, Dr. Rajendra Prasad Government Medical College and Hospital, Tanda, Himachal Pradesh, India. E-mail: seema 406@rediffmail.com 
Singla, et al.: The changing profile of infantile tremor syndrome

Table 1: Patient profile

\begin{tabular}{|c|c|c|c|c|c|c|c|}
\hline Patient number & Age (months) & Sex & Tremors & Lethargy & Neuroregression & Feeding & Diagnosis \\
\hline 1 & 18 & Female & - & ++ & + & Exclusive breast milk & Pre-ITS \\
\hline 2 & 18 & Male & ++ & +++ & +++ & Diluted buffalo milk & ITS \\
\hline 3 & 11 & Female & - & + & + & Exclusive breast milk & Pre-ITS \\
\hline 4 & 12 & Female & - & ++ & ++ & Exclusive breast milk & Pre-ITS \\
\hline 5 & 7 & Female & + & +++ & + & Exclusive breast milk & ITS \\
\hline 6 & 11 & Female & ++ & ++ & ++ & Diluted cow milk & ITS \\
\hline 7 & 18 & Female & ++ & ++ & +++ & Exclusive breast milk & ITS \\
\hline 8 & 11 & Male & + & +++ & + & Exclusive breast milk & ITS \\
\hline 9 & 16 & Female & - & ++ & ++ & Exclusive breast milk & Pre-ITS \\
\hline
\end{tabular}

Table 2: Clinical profile of patients of ITS

\begin{tabular}{lcc}
\hline Clinical features & Patients number & Patients (\%) \\
\hline Anemia & 09 & 100 \\
Macrocytic & 04 & 44 \\
Dimorphic & 04 & 44 \\
Microcytic & 01 & 11 \\
Tremors & 04 & 44 \\
Hypotonia & 02 & 22 \\
Hepatomegaly & 04 & 44 \\
Neuroregression & 08 & 89 \\
Splenomegaly & 02 & 22 \\
Urinary tract infection & 02 & 22 \\
LRTI & 01 & 11 \\
\hline
\end{tabular}

ITS: Infantile tremor syndrome, LRTI: Lower respiratory tract infection

All patients were hemodynamically stable at admission. All had severe pallor (100\%) with knuckle hyperpigmentation and sparse hair in most. Three (33\%) also had signs of rickets. Hepatomegaly was present in $4(44 \%)$, splenomegaly in $2(22 \%)$, and pneumonia in $1(11 \%)$.

Anemia (4-7.5 g/dl) was present in all. Peripheral blood film examination revealed the macrocytic picture in $4(44 \%)$, dimorphic in $4(44 \%)$, and microcytic in $1(11 \%)$. Chest X-rays had infiltrated in $3(33 \%)$ but workup for tubercular screening was negative. Sepsis screen was positive in 6 (66\%). Urine cultures were positive in $2(22 \%)$ with Escherichia coli and Pseudumonas in 1 each. Dyselectrolemia was present in 2 patients with hyponatremia in one and hypokalemia in other. Serum B12 levels were low in 4 out of 5 patients but could not be performed in all due to financial constraints. Renal and liver functions were normal in all the patients.

The mean duration of hospital stay was 9 days ranging from 4 to 16 days. Total 6 (66\%) with positive sepsis screen or cultures received antibiotics for the average duration of 9 days. Two also required blood transfusions. All signs and symptoms improved after treatment with supplements such as Vitamins A, D, K, B-complex, zinc, calcium, and magnesium along with dietary management. Propranolol was given to patients with ITS. On monthly follow-up, those with pre-ITS had better catch up in growth and development in all spheres. Some degree of developmental lag persisted in those with ITS.

\section{Discussion}

Infantile and pre-ITS is a well-known clinical entity reported primarily from the developing nations..$^{[4-6]}$ Despite its decreasing incidence, the finding of additional clinical features made us present this series. The etiology of ITS is still elusive. Among various theories, the nutritional theory is the most accepted. Vitamin B12 deficiency has been found to be associated with ITS in many studies. ${ }^{[1]}$ It is usually seen in children who are exclusively breastfed. Iron, magnesium, and zinc deficiency have also been postulated to cause ITS. Associated nutritional deficiencies such as protein, Vitamin A, D, C, and B-complex may be present. ${ }^{[7,8]}$ Other speculations for its etiology include viral encephalitis and degenerative processes..$^{[1,4,5]}$

Our series also found that all children with this syndrome had anemia, lethargy, and neuroregression and presented throughout the year with no seasonal clustering as previously stated. ${ }^{[4,6,9]}$ Furthermore, the type of anemia in this disease is not specific. On the other hand, it reflects the pattern of prevalence of the hematinic deficiencies in the region. However, unlike previous reports, there were more female patients, which may be due to bias in nutritional status.

The study adds the need for rational antibiotic use in patients of ITS, unlike many previous reports where all patients have been administered antibiotics. It reinforces the concept of sepsis screens and avoiding antibiotic abuse. The disease process may be superadded by urinary infections probably due to lower immunity state as a result of malnutrition, the leading cause of acquired immunodeficiency. It is also a reflection of changing epidemiology with no seasonal 
variation, showing the role of non-viral etiologies although previous studies show a seasonal pattern of disease. ${ }^{[10,11]}$ It highlights the need for better education for weaning practices and need for better community support.

\section{References}

1. Gupte S, Pal M, Gupta SK, Sangra KR. Infantile tremor syndrome (ITS). In: Gupte S, editor. Textbook of Paediatric Nutrition. New Delhi: Peepee; 2006. p. 255-65.

2. Holla CR, Prasad LC. Infantile tremor syndrome. Med J Armed Forces India 2010;66:186-7.

3. Agarwal R, Singh RN, Gupta BD, Agarwal DK, Arora AK. Serum magnesium, calcium, zinc in infantile tremor syndrome. Indian Pediatr 1993;30:374-6.

4. Ghai OP, Gupta P. Infantile tremor syndrome. In: Ghai OP, Gupta P,
Paul VK, editors. Ghai Essential Paediatrics. $8^{\text {th }}$ ed. New Delhi: Dr. Ghai; 2013. p. $580-1$.

5. Datta K, Datta S, Dutta I. Rare association of central pontinemyelinolysis with infantile tremor syndrome. Ann Indian Acad Neurol 2012;15:48-50.

6. Gupte S. Infantile tremor syndrome (ITS). Indian J Paediatr 2007;74:887.

7. Vora RM, Tullu MS, Bartakke SP, Kamat JR. Infantile tremor syndrome and zinc deficiency. Indian J Med Sci 2002;56:69-72.

8. Ratageri VH, Shepur TA, Patil MM, Hakeem MA. Scurvy in infantile tremor syndrome. Indian J Pediatr 2005;72:883-4.

9. Sharda B, Bhandari B. Infantile tremor syndrome. Indian Pediatr 1987;24:415-21

10. Goraya JS. Acute movement disorders in children: Experience from a developing country. J Child Neurol 2015;30:406-11.

11. Dikshit AK. Nutritional Dystrophy and anaemia. Indian J Child Health 1957;6:132-6.

How to cite this article: Singla DA, Sharma M, Sharma S, Sharma V. The changing profile of infantile tremor syndrome in hilly terrain of India. J Neurosci Rural Pract 2015;6:610-2.

Source of Support: Nil. Conflict of Interest: None declared. 